\title{
A Critical Analysis of Single Band Modeling of Thermoelectric Materials
}

\author{
Harshita Naithani ${ }^{*}$ and Titas Dasgupta** \\ Department of Metallurgical Engineering and Materials Science, Indian Institute of Technology \\ Bombay, Mumbai 400076, India.
}

KEYWORDS: Single Parabolic Band model (SPB), Single Kane Band model (SKB), thermoelectric materials, Seebeck coefficient, Lorenz number, band mass.

*- corresponding author. Email id- titas.dasgupta@iitb.ac.in 


\section{Solving the SPB and SKB equations using MATLAB}

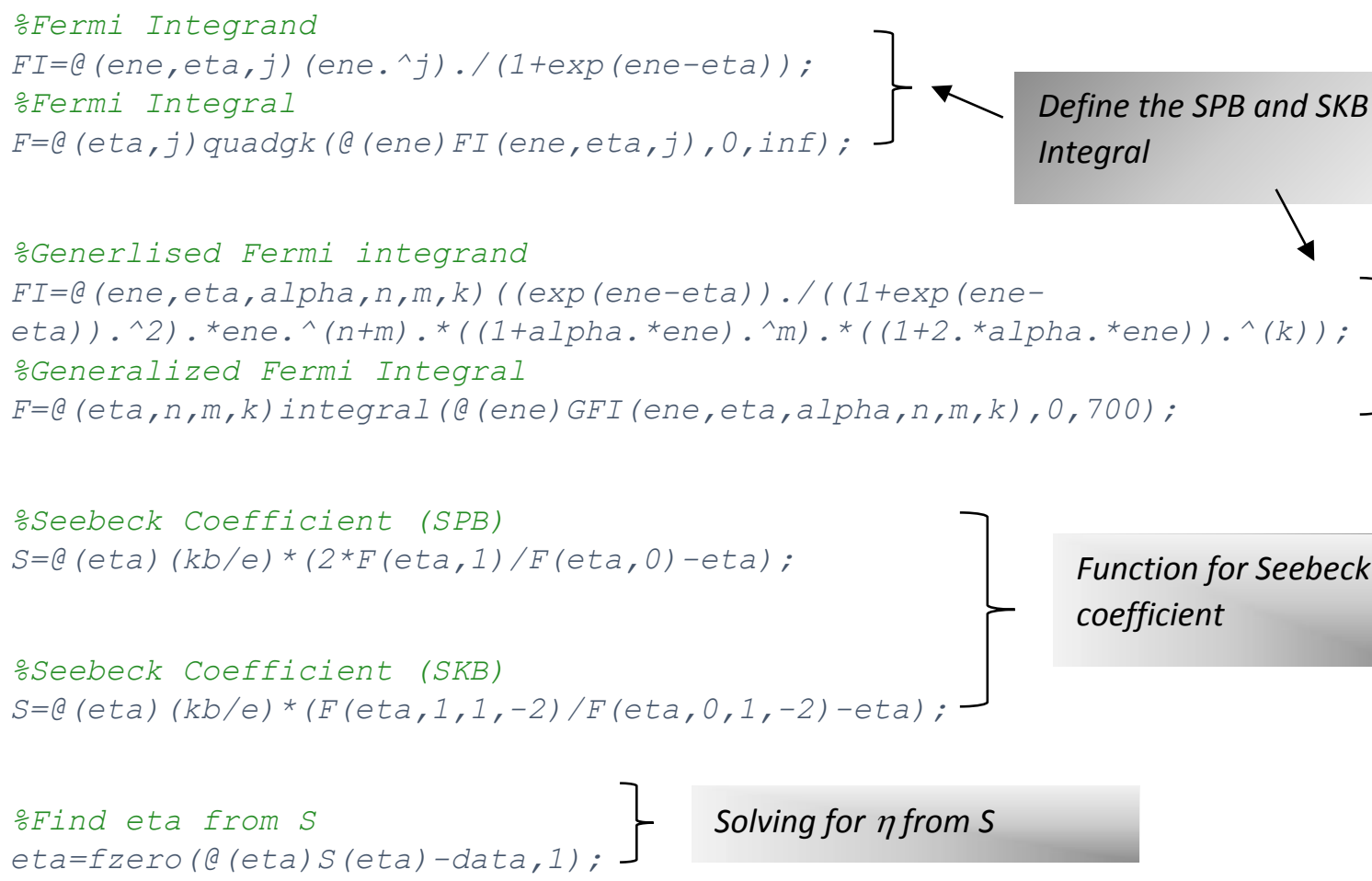

Here, ene represents the reduced energy ( $\varepsilon$ ), eta the reduced Fermi level ( $\eta)$, data is the experimental value of Seebeck coefficient

Figure S1. Representation of SPB/SKB equations using MATLAB. 
Table S1: Experimental data of the various materials used for the analysis.

\begin{tabular}{|c|c|c|c|c|}
\hline Composition & $S(\mu V / K)$ & $\sigma(S / \mathrm{cm})$ & $R_{H}\left(m^{-3}\right)$ & $\begin{array}{c}\text { Reference } \\
\text { no. }\end{array}$ \\
\hline $\mathrm{Mg}_{2.15} \mathrm{Si}_{0.28} \mathrm{Sn}_{0.71} \mathrm{Sb}_{0.006}$ & 158 & 1740 & $4.1 \mathrm{E}-08$ & 11 \\
\hline $\mathrm{Mg}_{2} \mathrm{Si}_{0.7} \mathrm{Sn}_{0.3}: \mathrm{Bi}_{0.01}$ & 461 & 7 & $1.3 \mathrm{E}-06$ & 12 \\
\hline $\mathrm{Mg}_{2}\left(\mathrm{Si}_{0.3} \mathrm{Sn} 0.7\right)_{1-\mathrm{y}} \mathrm{Bi}_{\mathrm{y}}$ & & & & 13 \\
\hline$y=0$ & 447 & 31 & $2.1 \mathrm{E}-06$ & \\
\hline 0.005 & 211 & 624 & $9.5 \mathrm{E}-08$ & \\
\hline 0.01 & 150 & 1533 & $3.8 \mathrm{E}-08$ & \\
\hline 0.015 & 130 & 2004 & $3.1 \mathrm{E}-08$ & \\
\hline 0.02 & 120 & 2328 & $2.8 \mathrm{E}-08$ & \\
\hline 0.025 & 115 & 2484 & $2.3 \mathrm{E}-08$ & \\
\hline 0.03 & 114 & 2672 & $2.3 \mathrm{E}-08$ & \\
\hline 0.04 & 103 & 2692 & $2.0 \mathrm{E}-08$ & \\
\hline $\mathrm{Mg}_{2}\left(\mathrm{Si}_{0.3} \mathrm{Sn}_{0.7}\right)_{0.98} \mathrm{Sb}_{0.02}$ & 115 & 2528 & $3.2 \mathrm{E}-08$ & 14 \\
\hline $\mathrm{Mg}_{2.2} \mathrm{Si}_{0.3} \mathrm{Sn}_{0.57} \mathrm{Sb}_{0.013}$ & 124 & 2110 & 1.7E-08 & 15 \\
\hline $\mathrm{Mg}_{2}\left(\mathrm{Sn}_{0.3} \mathrm{Sn}_{0.7}\right)_{0.98} \mathrm{Sb}_{0.02}$ & & & & 16 \\
\hline MS-PAS & 114 & 2947 & $2.5 \mathrm{E}-08$ & \\
\hline SSR-PAS & 120 & 2453 & $2.5 \mathrm{E}-08$ & \\
\hline MS-PAS-AN & 129 & 2294 & $2.8 \mathrm{E}-08$ & \\
\hline $\mathrm{Mg}_{2.10} \mathrm{Si}_{0.27} \mathrm{Sn}_{0.71} \mathrm{Sb}_{0.017}$ & 128 & 2345 & $2.9 \mathrm{E}-08$ & 17 \\
\hline $\mathrm{Mg}_{2.158}\left(\mathrm{Si}_{0.281} \mathrm{Sn}_{0.719}\right)_{0.978} \mathrm{Sb}_{0.022}$ & 125 & 2125 & $2.9 \mathrm{E}-08$ & 18 \\
\hline $\mathrm{Mg}_{2.148}\left(\mathrm{Si}_{0.289} \mathrm{Sn}_{0.711}\right)_{0.979} \mathrm{Sb}_{0.021}$ & 126 & 2034 & $3.1 \mathrm{E}-08$ & \\
\hline $\mathrm{Mg}_{2.139}\left(\mathrm{Si}_{0.306} \mathrm{Sn}_{0.694}\right)_{0.982} \mathrm{Sb}_{0.018}$ & 128 & 2032 & $3.2 \mathrm{E}-08$ & \\
\hline $\mathrm{Mg}_{2.082}\left(\mathrm{Si}_{0.346} \mathrm{Sn}_{0.654}\right)_{0.981} \mathrm{Sb}_{0.019}$ & 140 & 1868 & 3.7E-08 & \\
\hline $\mathrm{Mg}_{2} \mathrm{Si}_{0.3} \mathrm{Sn}_{0.7}$ & 381 & 83 & $7.9 \mathrm{E}-07$ & 19 \\
\hline $\mathrm{Mg}_{2}\left(\mathrm{Si}_{0.3} \mathrm{Sn}_{0.7}\right)_{0.98} \mathrm{Bi}_{0.02}$ & 113 & 2739 & $2.3 \mathrm{E}-08$ & \\
\hline $\mathrm{Mg}_{1.98} \mathrm{Cr}_{0.02}\left(\mathrm{Si}_{0.3} \mathrm{Sn}_{0.7}\right)_{0.98} \mathrm{Bi}_{0.02}$ & 129 & 2149 & $2.6 \mathrm{E}-08$ & \\
\hline $\mathrm{Mg}_{2}\left(\mathrm{Si}_{0.3} \mathrm{Sn}_{0.7}\right)_{1-\mathrm{y}} \mathrm{Sb}_{\mathrm{y}}$ & & & & 20 \\
\hline$y=.005$ & 176 & 1032 & $5.3 \mathrm{E}-08$ & \\
\hline$y=.01$ & 150 & 1506 & $3.6 \mathrm{E}-08$ & \\
\hline$y=.015$ & 125 & 2370 & $2.0 \mathrm{E}-08$ & \\
\hline$y=.02$ & 113 & 2586 & $1.7 \mathrm{E}-08$ & \\
\hline
\end{tabular}




\begin{tabular}{|c|c|c|c|c|}
\hline Composition & $S(\mu V / K)$ & $\sigma(S / \mathrm{cm})$ & $R_{H}\left(m^{-3}\right)$ & $\begin{array}{c}\text { Reference } \\
\text { no. }\end{array}$ \\
\hline$y=.025$ & 112 & 2536 & $1.9 \mathrm{E}-08$ & \\
\hline 3 & 76 & & $1.9 \mathrm{E}-08$ & 23 \\
\hline 5 & 91 & & 4.7E-08 & \\
\hline 8 & 95 & & $9.5 \mathrm{E}-08$ & \\
\hline 1 & 212 & & $1.2 \mathrm{E}-07$ & 24 \\
\hline 2 & 150 & 1515 & $3.3 \mathrm{E}-08$ & \\
\hline 3 & 142 & 1639 & $3.0 \mathrm{E}-08$ & \\
\hline 4 & 134 & 1515 & 2.7E-08 & \\
\hline 5 & 122 & 2174 & $2.3 \mathrm{E}-08$ & \\
\hline 6 & 117 & 2564 & $2.2 \mathrm{E}-08$ & \\
\hline 7 & 119 & 2273 & $1.8 \mathrm{E}-08$ & \\
\hline $\mathrm{Mg}_{2} \mathrm{Si}_{0.4-\mathrm{x}} \mathrm{Sn}_{0.6} \mathrm{Sb}_{\mathrm{x}}$ & & & & 25 \\
\hline$x=0$ & 647 & & $1.0 \mathrm{E}-05$ & \\
\hline$x=.0075$ & 156 & 1089 & $6.7 \mathrm{E}-08$ & \\
\hline$x=.015$ & 181 & 225 & 3.7E-07 & \\
\hline $\mathrm{Mg}_{2.16}\left(\mathrm{Si}_{0.4} \mathrm{Sn}_{0.6}\right)_{1-\mathrm{y}} \mathrm{Sb}_{\mathrm{y}}$ & & & & 26 \\
\hline$y=0$ & 412 & 25 & $3.2 \mathrm{E}-06$ & \\
\hline$y=.005$ & 190 & 600 & $1.1 \mathrm{E}-07$ & \\
\hline$y=.01$ & 143 & 1301 & 4.0E-08 & \\
\hline$y=.015$ & 132 & 1498 & 4.1E-08 & \\
\hline$y=.02$ & 116 & 1860 & $2.6 \mathrm{E}-08$ & \\
\hline$y=.025$ & 116 & 1845 & $2.9 \mathrm{E}-08$ & \\
\hline $\mathrm{Mg}_{2.16}\left(\mathrm{Si}_{0.4} \mathrm{Sn}_{0.6}\right)_{1-\mathrm{y}} \mathrm{Bi}_{\mathrm{y}}$ & & & & 27 \\
\hline$y=0$ & 413 & & $3.2 \mathrm{E}-06$ & \\
\hline 0.005 & 177 & 747 & $8.3 \mathrm{E}-08$ & \\
\hline 0.01 & 156 & 1279 & $4.8 \mathrm{E}-08$ & \\
\hline 0.015 & 136 & 1771 & $3.4 \mathrm{E}-08$ & \\
\hline 0.02 & 122 & 1854 & $2.9 \mathrm{E}-08$ & \\
\hline 0.03 & 122 & 2043 & $2.9 \mathrm{E}-08$ & \\
\hline $\mathrm{Mg}_{2.08} \mathrm{SiO}_{.4-\mathrm{x}} \mathrm{Sn}_{0.6} \mathrm{Sb}_{\mathrm{x}}(\mathrm{x})$ & & & & 28 \\
\hline 0 & 456 & 46 & $1.5 \mathrm{E}-06$ & \\
\hline 0.012 & 185 & 413 & $1.8 \mathrm{E}-07$ & \\
\hline 0.024 & 152 & 1021 & 3.3E-07 & \\
\hline 0.036 & 123 & 2583 & 2.7E-08 & \\
\hline 0.048 & 94 & 2535 & $1.1 \mathrm{E}-08$ & \\
\hline
\end{tabular}




\begin{tabular}{|c|c|c|c|c|}
\hline Composition & $S(\mu V / K)$ & $\sigma(S / \mathrm{cm})$ & $R_{H}\left(m^{-3}\right)$ & $\begin{array}{c}\text { Reference } \\
\text { no. }\end{array}$ \\
\hline 0.072 & 105 & 2160 & $1.1 \mathrm{E}-07$ & \\
\hline $\mathrm{Mg}_{2.08} \mathrm{Si}_{0.4-\mathrm{x}} \mathrm{Sn}_{0.6} \mathrm{Bi}_{\mathrm{x}}$ & & & & 29 \\
\hline 0 & 456 & 46 & $1.5 \mathrm{E}-06$ & \\
\hline 0.005 & 203 & 898 & & \\
\hline 0.01 & 150 & 1577 & $3.0 \mathrm{E}-08$ & \\
\hline 0.015 & 143 & 1820 & & \\
\hline 0.02 & 140 & 2013 & 2.7E-08 & \\
\hline 0.03 & 132 & 2286 & $2.1 \mathrm{E}-08$ & \\
\hline $\mathrm{Mg}_{2} \mathrm{Si}_{0.4} \mathrm{Sn}_{0.6-\mathrm{x}} \mathrm{Sb}_{\mathrm{x}}$ & & & & 30 \\
\hline 0.02 & 99 & 2009 & $3.0 \mathrm{E}-08$ & \\
\hline 0.11 & 273 & 119 & $8.9 \mathrm{E}-07$ & \\
\hline 0.12 & 90 & 2253 & $1.8 \mathrm{E}-08$ & \\
\hline 0.27 & 87 & 894 & 3.7E-08 & \\
\hline 0.38 & 84 & 405 & $6.0 \mathrm{E}-08$ & \\
\hline $\mathrm{Mg}_{2.24} \mathrm{Si}_{0.4} \mathrm{Sn}_{0.6}$ & 372 & 8 & $1.4 \mathrm{E}-06$ & 31 \\
\hline $\mathrm{Mg}_{2.24}\left(\mathrm{Si}_{0.4} \mathrm{Sn}_{0.6}\right)_{0.985} \mathrm{Sb}_{0.015}$ & 317 & 8 & $2.9 \mathrm{E}-06$ & \\
\hline $\mathrm{Mg}_{2.34} \mathrm{Si}_{0.4} \mathrm{Sn}_{0.6}$ & 286 & 60 & $1.1 \mathrm{E}-06$ & \\
\hline $\mathrm{Mg}_{2.34}\left(\mathrm{Si}_{0.4} \mathrm{Sn}_{0.6}\right)_{0.985} \mathrm{Sb}_{0.015}$ & 108 & 1721 & $2.2 \mathrm{E}-08$ & \\
\hline \multirow[t]{10}{*}{$\mathrm{YbxCO}_{4} \mathrm{Sb}_{12}$} & 321 & & $1.8 \mathrm{E}-06$ & 32 \\
\hline & 306 & & $1.1 \mathrm{E}-06$ & \\
\hline & 298 & & $8.6 \mathrm{E}-07$ & \\
\hline & 241 & & $3.0 \mathrm{E}-07$ & \\
\hline & 196 & & $1.4 \mathrm{E}-07$ & \\
\hline & 172 & & $8.5 \mathrm{E}-08$ & \\
\hline & 152 & & $5.0 \mathrm{E}-08$ & \\
\hline & 119 & & $2.2 \mathrm{E}-08$ & \\
\hline & 102 & & $1.5 \mathrm{E}-08$ & \\
\hline & 94 & & $1.2 \mathrm{E}-08$ & \\
\hline $\mathrm{CeFe}_{\mathrm{x}} \mathrm{Co}_{4-\mathrm{x}} \mathrm{Sb}_{12}(\mathrm{y}, \mathrm{x})$ & & & & 33 \\
\hline $.05,0$ & 185 & 535 & $1.2 \mathrm{E}-07$ & \\
\hline $.075,0$ & 153 & 935 & $4.9 \mathrm{E}-08$ & \\
\hline $.1,0$ & 176 & 1149 & $2.5 \mathrm{E}-08$ & \\
\hline $.15,0$ & 152 & 1316 & $2.9 \mathrm{E}-08$ & \\
\hline $.3, .75$ & 166 & 115 & $1.4 \mathrm{E}-08$ & \\
\hline $\mathrm{La}_{\mathrm{x}} \mathrm{Co}_{4} \mathrm{Sb}_{12}(\mathrm{x})$ & & & & 34 \\
\hline
\end{tabular}




\begin{tabular}{|c|c|c|c|c|}
\hline Composition & $S(\mu V / K)$ & $\sigma(S / \mathrm{cm})$ & $R_{H}\left(m^{-3}\right)$ & $\begin{array}{c}\text { Reference } \\
\text { no. }\end{array}$ \\
\hline 0.05 & 158 & 781 & $4.1 \mathrm{E}-08$ & \\
\hline 0.23 & 74 & 2128 & $3.2 \mathrm{E}-09$ & \\
\hline $\mathrm{Ba}_{0.3} \mathrm{Ni}_{\mathrm{x}} \mathrm{Co}_{4-\mathrm{x}} \mathrm{Sb}_{12}(\mathrm{x})$ & & & & 35 \\
\hline 0 & 97 & 1112 & $1.0 \mathrm{E}-08$ & \\
\hline 0.02 & 88 & 1499 & 7.6E-09 & \\
\hline 0.05 & 90 & 1942 & $6.0 \mathrm{E}-09$ & \\
\hline 0.08 & 91 & 2174 & $5.3 \mathrm{E}-09$ & \\
\hline 0.2 & 93 & 1600 & $3.6 \mathrm{E}-09$ & \\
\hline $\mathrm{Eu}_{\mathrm{x}} \mathrm{Co}_{4} \mathrm{Sb}_{12-\mathrm{y}} \mathrm{Ge}_{\mathrm{y}}(\mathrm{x}, \mathrm{y})$ & & & & 36 \\
\hline $.2,0$ & 174 & 1000 & 4.7E-08 & \\
\hline $.43, .31$ & 89 & 2941 & $7.2 \mathrm{E}-09$ & \\
\hline $.42, .5$ & 119 & 2000 & $1.1 \mathrm{E}-08$ & \\
\hline $\mathrm{Ca}_{\mathrm{x}} \mathrm{Co}_{4-\mathrm{y}} \mathrm{N}_{\mathrm{y}} \mathrm{Sb} 12(\mathrm{x}, \mathrm{y})$ & & & & 37 \\
\hline $.4,0$ & 111 & 400 & $2.2 \mathrm{E}-08$ & \\
\hline $.4, .02$ & 127 & 1010 & $2.0 \mathrm{E}-08$ & \\
\hline $.4, .05$ & 127 & 1887 & $1.4 \mathrm{E}-08$ & \\
\hline $.4, .08$ & 113 & 1563 & $1.2 \mathrm{E}-08$ & \\
\hline $\mathrm{Co}_{4} \mathrm{Sb}_{12-\mathrm{x}} \mathrm{Te}_{\mathrm{x}}(\mathrm{x})$ & & & & 38 \\
\hline 0.05 & 191 & 460 & $6.3 \mathrm{E}-08$ & \\
\hline 0.3 & 148 & 490 & $3.5 \mathrm{E}-08$ & \\
\hline 0.5 & 125 & 1000 & $1.1 \mathrm{E}-08$ & \\
\hline $\mathrm{Sr}_{\mathrm{y}} \mathrm{Co}_{4} \mathrm{Sb}_{12}(\mathrm{y})$ & & & & 39 \\
\hline 0.25 & 137 & 904 & $5.3 \mathrm{E}-08$ & \\
\hline 0.3 & 129 & 1355 & $4.3 \mathrm{E}-08$ & \\
\hline 0.35 & 119 & 1791 & $2.9 \mathrm{E}-08$ & \\
\hline 0.4 & 110 & 2717 & $1.8 \mathrm{E}-08$ & \\
\hline $\mathrm{Ca}_{y} \mathrm{Co}_{4-x} \mathrm{Ni}_{x} \mathrm{Sb}_{12}(\mathrm{y}, \mathrm{x})$ & & & & 40 \\
\hline $0.08,0$ & 148 & 333 & $2.0 \mathrm{E}-08$ & \\
\hline $.08, .02$ & 173 & 719 & $4.8 \mathrm{E}-08$ & \\
\hline $.1, .02$ & 167 & 680 & $3.9 \mathrm{E}-08$ & \\
\hline $.1, .05$ & 164 & 926 & $2.6 \mathrm{E}-08$ & \\
\hline $.2,0$ & 128 & 386 & $2.2 \mathrm{E}-08$ & \\
\hline $.15, .02$ & 135 & 1010 & $2.0 \mathrm{E}-08$ & \\
\hline $.18, .03$ & 133 & 1587 & $1.4 \mathrm{E}-08$ & \\
\hline $.18, .06$ & 127 & 1887 & $1.2 \mathrm{E}-08$ & \\
\hline
\end{tabular}




\begin{tabular}{|c|c|c|c|c|}
\hline Composition & $S(\mu V / K)$ & $\sigma(S / \mathrm{cm})$ & $R_{H}\left(m^{-3}\right)$ & $\begin{array}{c}\text { Reference } \\
\text { no. }\end{array}$ \\
\hline $\mathrm{Ba}_{\mathrm{x}} \mathrm{Yb}_{\mathrm{y}} \mathrm{Co}_{4} \mathrm{Sb}_{12}(\mathrm{x}, \mathrm{y})$ & & & & 41 \\
\hline $0.03,0$ & 220 & 399 & $1.4 \mathrm{E}-07$ & \\
\hline $.15, .01$ & 125 & 1798 & $2.4 \mathrm{E}-08$ & \\
\hline $.11, .03$ & 115 & 1787 & $1.8 \mathrm{E}-08$ & \\
\hline $0, .12$ & 146 & 765 & $3.0 \mathrm{E}-08$ & \\
\hline $.05, .09$ & 158 & 1126 & $2.4 \mathrm{E}-08$ & \\
\hline $.08, .09$ & 126 & 2068 & $2.1 \mathrm{E}-08$ & \\
\hline $.11, .08$ & 107 & 2114 & $1.5 \mathrm{E}-08$ & \\
\hline $\mathrm{Co}_{12} \mathrm{M}_{\mathrm{x}} \mathrm{Sb}_{3}(\mathrm{M})$ & & & & 42 \\
\hline \multirow[t]{3}{*}{$\mathrm{Pt}$} & 159 & & $1.0 \mathrm{E}-07$ & \\
\hline & 163 & & $6.9 \mathrm{E}-08$ & \\
\hline & 136 & & 4.3E-08 & \\
\hline \multirow[t]{3}{*}{$\mathrm{Ni}$} & 197 & & $6.0 \mathrm{E}-08$ & \\
\hline & 150 & & $2.4 \mathrm{E}-08$ & \\
\hline & 98 & & 6.7E-09 & \\
\hline \multirow[t]{4}{*}{$\mathrm{Pd}$} & 241 & & $2.0 \mathrm{E}-07$ & \\
\hline & 208 & & $1.1 \mathrm{E}-07$ & \\
\hline & 177 & & $5.0 \mathrm{E}-08$ & \\
\hline & 157 & & $3.3 \mathrm{E}-08$ & \\
\hline \multirow[t]{8}{*}{$\mathrm{Pd}+\mathrm{Pt}$} & 145 & & $4.0 \mathrm{E}-08$ & \\
\hline & 135 & & $3.4 \mathrm{E}-08$ & \\
\hline & 126 & & $2.5 \mathrm{E}-08$ & \\
\hline & 121 & & $2.1 \mathrm{E}-08$ & \\
\hline & 113 & & $2.4 \mathrm{E}-08$ & \\
\hline & 114 & & $2.0 \mathrm{E}-08$ & \\
\hline & 107 & & $1.8 \mathrm{E}-08$ & \\
\hline & 99 & & $1.4 \mathrm{E}-08$ & \\
\hline $\ln _{\mathrm{x}} \mathrm{Yb}_{\mathrm{y}} \mathrm{Co}_{4} \mathrm{Sb}_{12}(\mathrm{x}, \mathrm{y})$ & & & & 43 \\
\hline $.2,0$ & 236 & 532 & $1.2 \mathrm{E}-07$ & \\
\hline $.2, .05$ & 189 & 1316 & $5.6 \mathrm{E}-08$ & \\
\hline $.2, .2$ & 188 & 1389 & $5.3 \mathrm{E}-08$ & \\
\hline $.2, .2$ & 139 & 1587 & $2.4 \mathrm{E}-08$ & \\
\hline $0, .2$ & 168 & 357 & $3.4 \mathrm{E}-08$ & \\
\hline $.1, .1$ & 150 & 1316 & $3.4 \mathrm{E}-08$ & \\
\hline $\mathrm{Tl}_{\mathrm{x}} \mathrm{Co}_{4} \mathrm{Sb}_{12}(\mathrm{x})$ & & & & 44 \\
\hline
\end{tabular}




\begin{tabular}{|c|c|c|c|c|}
\hline Composition & $S(\mu V / K)$ & $\sigma(S / \mathrm{cm})$ & $R_{H}\left(m^{-3}\right)$ & $\begin{array}{c}\text { Reference } \\
\text { no. }\end{array}$ \\
\hline 0.1 & 185 & 741 & $1.1 \mathrm{E}-07$ & \\
\hline 0.22 & 125 & 1053 & 4.0E-08 & \\
\hline$\left(\mathrm{Sr}_{0.1} \mathrm{Yb}_{0.1}\right) \mathrm{Co}_{4} \mathrm{Sb}_{12}$ & 148 & 1062 & $4.3 \mathrm{E}-08$ & \\
\hline$\left(\mathrm{Ba}_{0.1} \mathrm{Yb}_{0.1}\right)_{0.75}\left(\mathrm{Sr}_{0.1} \mathrm{Yb}_{0.1}\right)_{0.25} \mathrm{Co}_{4} \mathrm{Sb}_{12}$ & 163 & 2358 & 4.9E-08 & \\
\hline$\left(\mathrm{Ba}_{0.1} \mathrm{Yb}_{0.1}\right)_{0.25}\left(\mathrm{Sr}_{0.1} \mathrm{Yb}_{0.1}\right)_{0.75} \mathrm{Co}_{4} \mathrm{Sb}_{12}$ & 157 & 2294 & $4.9 \mathrm{E}-08$ & \\
\hline$\left(\mathrm{Ba}_{0.1} \mathrm{Yb}_{0.1}\right)_{0.5}\left(\mathrm{Sr}_{0.1} \mathrm{Yb}_{0.1}\right)_{0.5} \mathrm{Co}_{4} \mathrm{Sb}_{12}$ & 157 & 2247 & $4.5 \mathrm{E}-08$ & \\
\hline$\left(\mathrm{Ba}_{0.5} \mathrm{Yb}_{0.5}\right)_{0.26} \mathrm{Co}_{4} \mathrm{Sb}_{12}$ & 127 & 2890 & $2.0 \mathrm{E}-08$ & \\
\hline$\left(\mathrm{Ba}_{0.1} \mathrm{Yb}_{0.1}\right) \mathrm{Co}_{4} \mathrm{Sb}_{12}$ & 155 & 1558 & $3.1 \mathrm{E}-08$ & \\
\hline$\left(\mathrm{Ba}_{0.5} \mathrm{Yb}_{0.5}\right)_{0.12} \mathrm{Co} 4 \mathrm{Sb} 12$ & 189 & 1404 & 4.7E-08 & \\
\hline $\mathrm{Ba}_{\mathrm{u}} \mathrm{La}_{\mathrm{v}} \mathrm{Yb}_{\mathrm{w}} \mathrm{Co}_{4} \mathrm{Sb}_{12}(\mathrm{u}, \mathrm{v}, \mathrm{w})$ & & & & 45 \\
\hline $.06, .05, .06$ & 138 & 1832 & $2.9 \mathrm{E}-08$ & \\
\hline $.08, .05, .08$ & 126 & 2398 & $1.9 \mathrm{E}-08$ & \\
\hline $.1, .05, .1$ & 107 & 3003 & $1.4 \mathrm{E}-08$ & \\
\hline $.1, .05, .15$ & 104 & 3058 & $1.2 \mathrm{E}-08$ & \\
\hline $.1, .05, .2$ & 93 & 3367 & 8.7E-09 & \\
\hline $\mathrm{Sr}_{0.16} \mathrm{Yb}_{0.03} \mathrm{Co}_{4} \mathrm{Sb}_{11.82}$ & 116 & 2980 & $1.5 \mathrm{E}-08$ & 46 \\
\hline $\mathrm{Sr}_{0.22} \mathrm{Yb}_{0.03} \mathrm{Co}_{4} \mathrm{Sb}_{12.12}$ & 109 & 3430 & $1.3 \mathrm{E}-08$ & \\
\hline annealing temperature & & & & 47 \\
\hline 673 & 213 & 301 & $3.6 \mathrm{E}-07$ & \\
\hline 773 & 275 & 228 & $5.8 \mathrm{E}-07$ & \\
\hline $\mathrm{Yb}_{0.2} \mathrm{Ce}_{0.15} \mathrm{Co}_{4} \mathrm{Sb}_{12}$ & 124 & 2123 & 1.9E-08 & 48 \\
\hline $\mathrm{Yb}_{0.2} \mathrm{In}_{0.2} \mathrm{Co}_{4} \mathrm{Sb}_{12}$ & 146 & 1827 & $2.4 \mathrm{E}-08$ & \\
\hline $\mathrm{Yb}_{0.2} \mathrm{In}_{0.2} \mathrm{Ce}_{0.15} \mathrm{Co}_{4} \mathrm{Sb}_{12}$ & 121 & 1943 & $1.2 \mathrm{E}-08$ & \\
\hline $\mathrm{Yb}_{0.3} \mathrm{In}_{0.2} \mathrm{Ce}_{0.15} \mathrm{Co}_{4} \mathrm{Sb}_{12}$ & 106 & 2547 & 1.0E-08 & \\
\hline $\mathrm{Ce}_{y} \mathrm{M}_{\mathrm{x}} \mathrm{Co}_{4-x} \mathrm{Sb}_{12}$ & & & & 49 \\
\hline $\mathrm{Ce}_{0.2} \mathrm{Co}_{4} \mathrm{Sb}_{12}$ & 145 & 1100 & $3.8 \mathrm{E}-08$ & \\
\hline $\mathrm{Ce}_{0.2} \mathrm{Co}_{3.95} \mathrm{Fe}_{0.05} \mathrm{Sb}_{12}$ & 120 & 1800 & $1.8 \mathrm{E}-08$ & \\
\hline $\mathrm{Ce}_{0.2} \mathrm{Co}_{3.9} \mathrm{Fe}_{0.1} \mathrm{Sb}_{12}$ & 134 & 1600 & $2.0 \mathrm{E}-08$ & \\
\hline $\mathrm{Ce}_{0.2} \mathrm{Co}_{3.975} \mathrm{Mn}_{0.025} \mathrm{Sb}_{12}$ & 127 & 1900 & $2.1 \mathrm{E}-08$ & \\
\hline $\mathrm{Yb}_{0.08} \mathrm{Ba}_{0.09} \mathrm{Co}_{4} \mathrm{Sb}_{12.12} / \mathrm{SQ}$ & 128 & 1786 & $2.6 \mathrm{E}-08$ & 50 \\
\hline $\mathrm{Yb}_{0.08} \mathrm{Ba}_{0.09} \mathrm{Co}_{4} \mathrm{Sb}_{12.12} / \mathrm{FQ}$ & 127 & 1852 & $2.6 \mathrm{E}-08$ & \\
\hline $\mathrm{Yb}_{0.13} \mathrm{Ba}_{0.10} \mathrm{Co}_{4} \mathrm{Sb}_{12} / \mathrm{SQ}$ & 122 & 2439 & $1.8 \mathrm{E}-08$ & \\
\hline $\mathrm{Yb}_{0.13} \mathrm{Ba}_{0.10} \mathrm{Co}_{4} \mathrm{Sb}_{12} / \mathrm{FQ}$ & 117 & 2174 & $1.9 \mathrm{E}-08$ & \\
\hline $\mathrm{Yb}_{0.13} \mathrm{Ba}_{0.10} \mathrm{Co}_{4} \mathrm{Sb}_{12} / \mathrm{VFQ}$ & 114 & 2564 & $1.5 \mathrm{E}-08$ & \\
\hline
\end{tabular}




\begin{tabular}{|c|c|c|c|c|}
\hline Composition & $S(\mu V / K)$ & $\sigma(S / \mathrm{cm})$ & $R_{H}\left(m^{-3}\right)$ & $\begin{array}{c}\text { Reference } \\
\text { no. }\end{array}$ \\
\hline $\mathrm{Yb}_{2} \mathrm{Co}_{4} \mathrm{Sb}_{12}(321 \mathrm{~K})$ & & & & 51 \\
\hline 0.1 & 192 & 276 & $4.1 \mathrm{E}-08$ & \\
\hline 0.2 & 155 & 1539 & $2.5 \mathrm{E}-08$ & \\
\hline 0.3 & 124 & 2053 & $1.4 \mathrm{E}-08$ & \\
\hline 0.4 & 117 & 2103 & $8.6 \mathrm{E}-09$ & \\
\hline $\mathrm{Ce}_{y} \mathrm{Co}_{4-x} \mathrm{Cr}_{x} \mathrm{Sb}_{12}(\mathrm{y}, \mathrm{x})$ & & & & 52 \\
\hline $.2,0$ & 160 & 1035 & $3.3 \mathrm{E}-08$ & \\
\hline $.2, .025$ & 160 & 1096 & $3.3 \mathrm{E}-08$ & \\
\hline $.2, .05$ & 145 & 1346 & $2.1 \mathrm{E}-08$ & \\
\hline $.2, .1$ & 170 & 641 & $3.1 \mathrm{E}-08$ & \\
\hline $.25,0$ & 151 & 1413 & $3.0 \mathrm{E}-08$ & \\
\hline $.25,0.025$ & 152 & 1437 & $2.7 \mathrm{E}-08$ & \\
\hline $.25, .05$ & 149 & 1384 & $2.3 \mathrm{E}-08$ & \\
\hline $.25, .1$ & 168 & 1081 & $2.3 \mathrm{E}-08$ & \\
\hline $\operatorname{CoSb}_{2.9} \mathrm{Se}_{0.1}$ & 236 & 119 & $1.2 \mathrm{E}-07$ & 53 \\
\hline $\operatorname{CoSb}_{2.825} \mathrm{Te}_{0.15} \mathrm{Se}_{.025}$ & 115 & 1136 & $1.5 \mathrm{E}-08$ & \\
\hline $\mathrm{CoSb}_{2.8} \mathrm{Te}_{0.15} \mathrm{Se}_{0.05}$ & 142 & 1111 & $2.0 \mathrm{E}-08$ & \\
\hline $\mathrm{CoSb}_{2.75} \mathrm{Te}_{0.15} \mathrm{Se}_{0.1}$ & 126 & 885 & $1.8 \mathrm{E}-08$ & \\
\hline $\mathrm{Ca}_{\mathrm{x}} \mathrm{CO}_{4} \mathrm{Sb}_{12}(\mathrm{x})$ & & & & 54 \\
\hline 0.2 & 160 & 1285 & $3.2 \mathrm{E}-08$ & \\
\hline 1 & 103 & 3226 & $1.2 \mathrm{E}-08$ & \\
\hline \multicolumn{5}{|l|}{$\mathrm{La}_{\mathrm{x}} \mathrm{Co}_{4} \mathrm{Sb}_{12}(\mathrm{x})$} \\
\hline 0.3 & 164 & 498 & $7.0 \mathrm{E}-08$ & 55 \\
\hline 0.5 & 140 & 1163 & $2.9 \mathrm{E}-08$ & \\
\hline 0.7 & 109 & 2326 & $1.1 \mathrm{E}-08$ & \\
\hline $\mathrm{ZnSb}$ & & & & 56 \\
\hline undoped & 385 & 10 & & \\
\hline .05 at\% Sn & 278 & 54 & $3.5 \mathrm{E}-06$ & \\
\hline .5 at\% Sn & 236 & 97 & $1.6 \mathrm{E}-06$ & \\
\hline \multirow[t]{6}{*}{ Individual Samples } & 452 & & $3.3 \mathrm{E}-05$ & \\
\hline & 437 & & $4.6 \mathrm{E}-05$ & \\
\hline & 391 & & $7.5 \mathrm{E}-06$ & \\
\hline & 389 & & $1.7 \mathrm{E}-05$ & \\
\hline & 377 & & $2.0 \mathrm{E}-05$ & \\
\hline & 372 & & $1.7 \mathrm{E}-05$ & \\
\hline
\end{tabular}




\begin{tabular}{|c|c|c|c|c|}
\hline Composition & $S(\mu V / K)$ & $\sigma(S / \mathrm{cm})$ & $R_{H}\left(m^{-3}\right)$ & $\begin{array}{c}\text { Reference } \\
\text { no. }\end{array}$ \\
\hline & 363 & & $1.7 \mathrm{E}-05$ & \\
\hline & 351 & & $1.9 \mathrm{E}-05$ & \\
\hline & 369 & & $9.1 \mathrm{E}-06$ & \\
\hline & 359 & & $7.8 \mathrm{E}-06$ & \\
\hline & 347 & & $6.1 \mathrm{E}-06$ & \\
\hline & 330 & & $8.0 \mathrm{E}-06$ & \\
\hline & 318 & & $8.2 \mathrm{E}-06$ & \\
\hline & 303 & & $7.3 \mathrm{E}-06$ & \\
\hline & 296 & & $7.3 \mathrm{E}-06$ & \\
\hline & 279 & & $6.6 \mathrm{E}-06$ & \\
\hline & 302 & & $5.2 \mathrm{E}-06$ & \\
\hline & 318 & & $4.0 \mathrm{E}-06$ & \\
\hline & 317 & & $3.8 \mathrm{E}-06$ & \\
\hline & 273 & & $1.9 \mathrm{E}-06$ & \\
\hline & 260 & & $1.8 \mathrm{E}-06$ & \\
\hline & 247 & & $1.6 \mathrm{E}-06$ & \\
\hline & 255 & & $1.6 \mathrm{E}-06$ & \\
\hline & 244 & & $1.2 \mathrm{E}-06$ & \\
\hline & 221 & & $1.1 \mathrm{E}-06$ & \\
\hline & 226 & & $9.6 \mathrm{E}-07$ & \\
\hline $\mathrm{Zn}_{3} \mathrm{P}_{2}$ at\% (333K) & & & & 57 \\
\hline 0 & 325 & 40 & $5.2 \mathrm{E}-06$ & \\
\hline 0.625 & 362 & 21 & $1.3 \mathrm{E}-05$ & \\
\hline 1.5 & 408 & 24 & $1.0 \mathrm{E}-05$ & \\
\hline 2.5 & 393 & 26 & $8.2 \mathrm{E}-06$ & \\
\hline 3.75 & 381 & 22 & $1.4 \mathrm{E}-05$ & \\
\hline \multicolumn{5}{|c|}{$\mathrm{Zn}_{3} \mathrm{P}_{2}$ at $\%, \mathrm{Cu}$ at $\%(313 \mathrm{~K})$} \\
\hline $2.5,0.05$ & 175 & 204 & $1.2 \mathrm{E}-06$ & \\
\hline $2.5,0.1$ & 148 & 500 & $3.8 \mathrm{E}-07$ & \\
\hline $2.5,0.2$ & 137 & 714 & $3.4 \mathrm{E}-07$ & \\
\hline $\mathrm{Zn}_{1-x} \mathrm{Ag}_{\mathrm{x}} \mathrm{Sb}$ & & & & 58 \\
\hline$x=0$ & 284 & & $5.2 \mathrm{E}-06$ & \\
\hline$x=.002$ & 181 & & $2.4 \mathrm{E}-07$ & \\
\hline$x=.02$ & 171 & & $1.6 \mathrm{E}-07$ & \\
\hline Cu at \% & & & & 59 \\
\hline 0.6 & 134 & 640 & $2.5 \mathrm{E}-07$ & \\
\hline 0.3 & 140 & 570 & $2.3 \mathrm{E}-07$ & \\
\hline 0.15 & 142 & 935 & $2.6 \mathrm{E}-07$ & \\
\hline
\end{tabular}




\begin{tabular}{|c|c|c|c|c|}
\hline Composition & $S(\mu V / K)$ & $\sigma(S / \mathrm{cm})$ & $R_{H}\left(m^{-3}\right)$ & $\begin{array}{c}\text { Reference } \\
\text { no. }\end{array}$ \\
\hline 0.1 & 158 & 770 & 3.7E-07 & \\
\hline \multicolumn{5}{|l|}{ CuSb at \% } \\
\hline 0.3 & 129 & 830 & $2.6 \mathrm{E}-07$ & \\
\hline 0.6 & 130 & 885 & $2.7 \mathrm{E}-07$ & \\
\hline Cu at\% & & & & 60 \\
\hline 0.05 & 203 & 403 & 5.7E-07 & \\
\hline 0 & 305 & 110 & $4.8 \mathrm{E}-06$ & \\
\hline at\% Cu (322K) & & & & 61 \\
\hline 0 & 333 & 53 & $5.2 \mathrm{E}-06$ & \\
\hline 0.025 & 255 & 175 & $1.2 \mathrm{E}-06$ & \\
\hline 0.05 & 246 & 304 & $9.0 \mathrm{E}-07$ & \\
\hline 0.075 & 213 & 382 & $5.6 \mathrm{E}-07$ & \\
\hline 0.1 & 198 & 522 & $4.3 \mathrm{E}-07$ & \\
\hline 0.2 & 190 & 587 & $4.0 \mathrm{E}-07$ & \\
\hline 0.3 & 190 & 484 & $3.8 \mathrm{E}-07$ & \\
\hline \multicolumn{5}{|c|}{ Cu at \%and $0.2 \% \mathrm{P}(323 \mathrm{~K})$} \\
\hline 0 & 283 & 56 & $3.4 \mathrm{E}-06$ & \\
\hline 0.05 & 221 & 117 & $1.6 \mathrm{E}-06$ & \\
\hline 0.1 & 239 & 205 & $7.4 \mathrm{E}-07$ & \\
\hline 0.2 & 188 & 282 & $5.3 \mathrm{E}-07$ & \\
\hline 0.3 & 170 & 336 & $4.1 \mathrm{E}-07$ & \\
\hline 0.4 & 145 & 561 & $3.8 \mathrm{E}-07$ & \\
\hline $\mathrm{Zn}_{1-\mathrm{x}} \mathrm{Sb}(\% \mathrm{x})$ & & & & 62 \\
\hline 0 & 399 & 5 & $1.8 \mathrm{E}-05$ & \\
\hline 1 & 345 & 6 & $1.4 \mathrm{E}-05$ & \\
\hline 2 & 281 & 7 & 1.1E-05 & \\
\hline 3 & 266 & 8 & $9.5 \mathrm{E}-06$ & \\
\hline 4 & 263 & 8 & $9.4 \mathrm{E}-06$ & \\
\hline$\% \mathrm{Sn}$ & & & & 63 \\
\hline 0.1 & 169 & 470 & $4.6 \mathrm{E}-07$ & \\
\hline 0.2 & 194 & 314 & 5.7E-07 & \\
\hline 0.5 & 295 & 107 & $3.0 \mathrm{E}-06$ & \\
\hline \multicolumn{5}{|l|}{$\% Z n S n$} \\
\hline 0.1 & 238 & 192 & $1.2 \mathrm{E}-06$ & \\
\hline 0.2 & 222 & 243 & 8.7E-07 & \\
\hline 0.5 & 194 & 252 & $7.6 \mathrm{E}-07$ & \\
\hline
\end{tabular}




\begin{tabular}{|c|c|c|c|c|}
\hline Composition & $S(\mu V / K)$ & $\sigma(S / \mathrm{cm})$ & $R_{H}\left(m^{-3}\right)$ & $\begin{array}{c}\text { Reference } \\
\text { no. }\end{array}$ \\
\hline LP & 456 & 12 & $2.1 \mathrm{E}-05$ & 64 \\
\hline \multirow[t]{12}{*}{ MA } & 340 & 59 & $4.9 \mathrm{E}-06$ & \\
\hline & 250 & 139 & & 65 \\
\hline & 210 & 526 & & \\
\hline & 490 & 20 & $2.3 \mathrm{E}-05$ & \\
\hline & 375 & 20 & $5.6 \mathrm{E}-06$ & \\
\hline & 404 & 25 & 1.7E-05 & \\
\hline & 288 & 33 & $9.4 \mathrm{E}-06$ & \\
\hline & 400 & 50 & $8.8 \mathrm{E}-06$ & \\
\hline & 181 & 625 & 2.7E-07 & \\
\hline & 250 & & $1.5 \mathrm{E}-06$ & \\
\hline & 134 & & 2.7E-07 & \\
\hline & 142 & & 2.9E-07 & \\
\hline $\mathrm{Ag} 0.04 \mathrm{PbTe}+\mathrm{x} \%$ & & & & 67 \\
\hline 1 & 299 & 119 & $6.1 \mathrm{E}-06$ & \\
\hline 2 & 315 & 97 & 7.3E-06 & \\
\hline 3 & 323 & 74 & $8.4 \mathrm{E}-06$ & \\
\hline 4 & 314 & 91 & 7.1E-06 & \\
\hline 0.04 & 316 & 82 & 7.7E-06 & \\
\hline $\operatorname{PbTe}_{(1-x)} l_{x}$ & & & & 68 \\
\hline 0.0004 & 149 & 1613 & $1.1 \mathrm{E}-06$ & \\
\hline 0.0007 & 117 & 2564 & $6.3 \mathrm{E}-07$ & \\
\hline 0.0012 & 83 & 4255 & $3.7 \mathrm{E}-07$ & \\
\hline 0.002 & 65 & 7813 & $2.2 \mathrm{E}-07$ & \\
\hline 0.0028 & 50 & 8333 & $1.5 \mathrm{E}-07$ & \\
\hline 0.004 & 31 & 11001 & $1.1 \mathrm{E}-07$ & \\
\hline 0.0055 & 25 & 11001 & $7.9 \mathrm{E}-08$ & \\
\hline 0.01 & 25 & 11001 & 4.3E-08 & \\
\hline & & & & \\
\hline & & & & 60 \\
\hline I1 & 81 & 3846 & $3.5 \mathrm{E}-07$ & \\
\hline 12 & 60 & 4950 & $2.2 \mathrm{E}-07$ & \\
\hline La1 & 90 & 2564 & $3.5 \mathrm{E}-07$ & \\
\hline La2 & 70 & 3268 & $2.0 \mathrm{E}-07$ & \\
\hline
\end{tabular}




\begin{tabular}{|c|c|c|c|c|}
\hline Composition & $S(\mu V / K)$ & $\sigma(S / \mathrm{cm})$ & $R_{H}\left(m^{-3}\right)$ & $\begin{array}{c}\text { Reference } \\
\text { no. }\end{array}$ \\
\hline \multicolumn{5}{|l|}{ Pisa Data } \\
\hline \multirow[t]{30}{*}{1} & 175 & & $1.2 \mathrm{E}-06$ & \\
\hline & 145 & & $1.2 \mathrm{E}-06$ & \\
\hline & 150 & & $1.0 \mathrm{E}-06$ & \\
\hline & 130 & & $6.9 \mathrm{E}-07$ & \\
\hline & 124 & & $6.1 \mathrm{E}-07$ & \\
\hline & 114 & & 5.7E-07 & \\
\hline & 109 & & $6.3 \mathrm{E}-07$ & \\
\hline & 104 & & $6.1 \mathrm{E}-07$ & \\
\hline & 96 & & 4.1E-07 & \\
\hline & 90 & & $4.2 \mathrm{E}-07$ & \\
\hline & 79 & & 3.5E-07 & \\
\hline & 74 & & $3.1 \mathrm{E}-07$ & \\
\hline & 66 & & 3.1E-07 & \\
\hline & 71 & & 2.7E-07 & \\
\hline & 75 & & 2.7E-07 & \\
\hline & 66 & & $2.2 \mathrm{E}-07$ & \\
\hline & 60 & & $2.2 \mathrm{E}-07$ & \\
\hline & 58 & & 2.0E-07 & \\
\hline & 55 & & $1.8 \mathrm{E}-07$ & \\
\hline & 50 & & $1.5 \mathrm{E}-07$ & \\
\hline & 40 & & $1.4 \mathrm{E}-07$ & \\
\hline & 42 & & $1.2 \mathrm{E}-07$ & \\
\hline & 38 & & $1.1 \mathrm{E}-07$ & \\
\hline & 30 & & $1.2 \mathrm{E}-07$ & \\
\hline & 31 & & 1.1E-07 & \\
\hline & 34 & & 9.9E-08 & \\
\hline & 28 & & 7.7E-08 & \\
\hline & 21 & & 5.6E-08 & \\
\hline & 21 & & $5.2 \mathrm{E}-08$ & \\
\hline & 24 & & 4.3E-08 & \\
\hline \multirow[t]{9}{*}{ La } & 170 & & 1.5E-06 & \\
\hline & 124 & & $6.5 \mathrm{E}-07$ & \\
\hline & 90 & & 3.3E-07 & \\
\hline & 73 & & $2.3 \mathrm{E}-07$ & \\
\hline & 70 & & $2.0 \mathrm{E}-07$ & \\
\hline & 71 & & $1.8 \mathrm{E}-07$ & \\
\hline & 55 & & $1.5 \mathrm{E}-07$ & \\
\hline & 50 & & 1.2E-07 & \\
\hline & 53 & & $1.1 \mathrm{E}-07$ & \\
\hline
\end{tabular}




\begin{tabular}{|c|c|c|c|c|}
\hline Composition & $S(\mu V / K)$ & $\sigma(S / \mathrm{cm})$ & $R_{H}\left(m^{-3}\right)$ & $\begin{array}{c}\text { Reference } \\
\text { no. }\end{array}$ \\
\hline & 47 & & $1.0 \mathrm{E}-07$ & \\
\hline & 40 & & $6.7 \mathrm{E}-08$ & \\
\hline & 42 & & $5.8 \mathrm{E}-08$ & \\
\hline \multirow[t]{8}{*}{$\mathrm{Pb}_{1-\mathrm{x}} \mathrm{La}_{\mathrm{x}} \mathrm{Te}$} & 171 & & $1.5 \mathrm{E}-06$ & 70 \\
\hline & 126 & & $6.4 \mathrm{E}-07$ & \\
\hline & 93 & & 3.3E-07 & \\
\hline & 76 & & $2.7 \mathrm{E}-07$ & \\
\hline & 72 & & $2.0 \mathrm{E}-07$ & \\
\hline & 72 & & $1.7 \mathrm{E}-07$ & \\
\hline & 54 & & $1.1 \mathrm{E}-07$ & \\
\hline & 41 & & $6.6 \mathrm{E}-08$ & \\
\hline at. $\%$ & & & & 71 \\
\hline 0.3 & 320 & 151 & $4.9 \mathrm{E}-06$ & \\
\hline 0.4 & 240 & 473 & $2.2 \mathrm{E}-06$ & \\
\hline 0.6 & 151 & 1420 & $8.6 \mathrm{E}-07$ & \\
\hline 0.8 & 74 & 3160 & $3.0 \mathrm{E}-07$ & \\
\hline 30 & 69 & 3365 & $1.4 \mathrm{E}-07$ & 72 \\
\hline $3 R$ & 73 & 2952 & $1.5 \mathrm{E}-07$ & \\
\hline 35 & 57 & 5213 & $1.3 \mathrm{E}-07$ & \\
\hline $3 T$ & 44 & 6748 & $1.1 \mathrm{E}-07$ & \\
\hline $2 \mathrm{~V}$ & 42 & 6962 & $1.1 \mathrm{E}-07$ & \\
\hline $\mathrm{Pb}_{(1-\mathrm{x})} \mathrm{Al}_{\mathrm{x}} \mathrm{Te}(\% \mathrm{x})$ & & & & 73 \\
\hline 0.125 & 117 & & $5.2 \mathrm{E}-07$ & \\
\hline 0.25 & 82 & & $2.7 \mathrm{E}-07$ & \\
\hline 0.5 & 51 & & $1.6 \mathrm{E}-07$ & \\
\hline 1 & 57 & & $1.6 \mathrm{E}-07$ & \\
\hline $\mathrm{Pb}_{(1-\mathrm{x})} \mathrm{Cr}_{\mathrm{x}} \mathrm{Te}(\% \mathrm{x})$ & & & & 74 \\
\hline 0.25 & 185 & & $1.3 \mathrm{E}-06$ & \\
\hline 0.5 & 175 & & $1.2 \mathrm{E}-06$ & \\
\hline 1 & 159 & & $9.9 \mathrm{E}-07$ & \\
\hline 2 & 152 & & $8.5 \mathrm{E}-07$ & \\
\hline & & & & 75 \\
\hline $0.5 \% \mathrm{Cl}$ & 59 & & $1.7 \mathrm{E}-07$ & \\
\hline $.3 \% \mathrm{Cl}$ & 89 & & $3.0 \mathrm{E}-07$ & \\
\hline
\end{tabular}




\begin{tabular}{|l|c|c|c|c|}
\hline Composition & $\boldsymbol{S}(\boldsymbol{\mu} \mathbf{V} / \boldsymbol{K})$ & $\sigma(\mathrm{S} / \mathrm{cm})$ & $\boldsymbol{R}_{\boldsymbol{H}}\left(\mathbf{m}^{-\mathbf{3}}\right)$ & $\begin{array}{c}\text { Reference } \\
\text { no. }\end{array}$ \\
\hline $.3 \% \mathrm{NaCl}$ & 119 & & $1.0 \mathrm{E}-06$ & \\
\hline $.1 \% \mathrm{NaCl}$ & 148 & & $1.4 \mathrm{E}-06$ & \\
\hline & & & & \\
\hline $\mathrm{Pb}_{(1-\mathrm{-})} \mathrm{Bi}_{\mathrm{x}} \mathrm{Te}$ & & & & 76 \\
\hline 0.005 & 101 & 661 & $6.9 \mathrm{E}-07$ & \\
\hline 0.01 & 91 & 1410 & $4.5 \mathrm{E}-07$ & \\
\hline 0.02 & 86 & 2006 & $2.6 \mathrm{E}-07$ & \\
\hline 0.05 & 72 & 2528 & $1.1 \mathrm{E}-07$ & \\
\hline
\end{tabular}

Table S2: Parameters used for the two band model

\begin{tabular}{|l|l|l|l|l|l|}
\hline Material & $m_{d 1}^{*}\left(V m_{e}\right)$ & $m_{d 2}^{*}\left(m_{e}\right)$ & $\mu_{01}\left(m^{2} / V s\right)$ & $\mu_{02}\left(m^{2} / V s\right)$ & $\begin{array}{l}\text { Interband } \\
\text { Separation (eV) }\end{array}$ \\
\hline $\mathrm{Mg}_{2} \mathrm{Si}_{0.3} \mathrm{Sn}_{0.7}$ & 1.1 & 2.19 & 0.0123 & 0.0078 & 0.00 \\
\hline $\mathrm{Mg}_{2} \mathrm{Si}_{0.4} \mathrm{Sn}_{0.6}$ & 1.1 & 2.19 & 0.0123 & 0.0078 & 0.06 \\
\hline $\mathrm{CoSb}_{3}$ & 0.7 & 4.8 & 0.0147 & 0.0040 & 0.08 \\
\hline $\mathrm{ZnSb}$ & 0.2 & 0.8 & 0.0276 & 0.0281 & 0.08 \\
\hline
\end{tabular}

Table S3: Parameters used for SKB model

\begin{tabular}{|l|l|l|}
\hline Material & $\mathrm{K}$ & $\mathrm{E}_{\mathrm{G}}(\mathrm{eV})$ \\
\hline Mg2Si0.3Sn0.7 & 1 & 0.39 \\
\hline Mg2Si0.4Sn0.6 & 1 & 0.44 \\
\hline CoSb3 & 5 & 0.22 \\
\hline $\mathrm{ZnSb}$ & 1.35 & 0.35 \\
\hline $\mathrm{PbTe}$ & 3.6 & 0.30 \\
\hline
\end{tabular}




\section{Procedure for calculating $\mathrm{m}_{\mathrm{D}}^{*}$ av and $\mu_{0}{ }^{\mathrm{av}}$}

Following procedure has been used to calculate $m^{*}{ }^{\text {av }}$ :

1. Experimental values of Seebeck coefficient $\left(\mathrm{S}_{\text {exp }}\right)$ and corresponding charge carrier concentration (n) values (obtained using single band model equations) were taken as input.

2. For a guess value of $\mathrm{m}_{\mathrm{D}}^{*}$, Reduced Fermi level $(\eta)$ value was calculated for $i^{\text {th }} \mathrm{n}$ using (here $i$ represents a set of $S, n$ data)

$$
n=\frac{\left(2 m_{D}^{*} k_{B} T\right)^{3 / 2}}{3 \pi^{2} \hbar^{3}} F_{1 / 2}(\eta)
$$

3. Using that $\eta$, theoretical $S\left(S_{\text {model }}\right)$ value was obtained.

$$
S_{\text {model }}=\frac{k_{B}}{e}\left(\frac{2 F_{1}(\eta)}{F_{0}(\eta)}-\eta\right)
$$

4. $\left(S_{\text {exp }}-S_{\text {model }}\right)^{2}$ was obtained.

5. Steps 2-4 were repeated for all input $\mathrm{S}$ and $\mathrm{n}$ (all $i$ values).

6. S least square error $=\sum_{i}\left(S_{\text {exp }}-S_{\text {model }}\right)^{2}$ was obtained.

7. This was done for different guess values of $m^{*}$ and the one corresponding to the minimum $S$ least square error was taken as $m^{*}{ }^{a v}$.

8. $\mathrm{S}_{\text {rms }}^{\text {error }}$ value is given by $\sqrt{\frac{\sum_{i=1}^{N}\left(S_{\text {exp }}-S_{\text {model }}\right)^{2}}{N}}$, with $\mathrm{S}_{\text {model }}$ calculated using $\mathrm{m}_{\mathrm{D}}^{*}{ }_{\mathrm{D}}^{\text {av }}$ value.

These steps were repeated with SKB equations for obtaining the corresponding $\mathrm{m}_{\mathrm{D}}{ }^{\mathrm{av}}$.

For $\mu_{0}^{\mathrm{av}}$ :

1. $\mathrm{n}$ and $\mu_{\exp }$ (mobility) obtained from single band models along with $\mathrm{m}^{*}{ }_{\mathrm{D}}^{\mathrm{av}}$ were taken as input.

2. Reduced Fermi level ( $\eta$ ) was calculated for $i^{\text {th }} \mathrm{n}$.

3. Hall prefactor was calculated using $\eta$.

4. For a guess value of $\mu_{0}, \mu_{\text {model }}$ was calculated.

5. Steps 2-4 were repeated for all input $\mathrm{n}$ and $\mu$.

6. $\mu$ least square error $=\sum_{i}\left(\mu_{\text {exp }}-\mu_{\text {model }}\right)^{2}$ was obtained.

7. This was done for different guess values of $\mu_{0}$ and the one corresponding to the minimum $\mu$ least square error was taken as $\mu_{0}{ }^{\text {av }}$. 
Table S4: The $\mathrm{m}_{\mathrm{D}}^{*}$ av and $\mu_{0}{ }^{a v}$ values obtained from the least square fitting.

\begin{tabular}{|l|l|l|l|l|}
\hline \multirow{2}{*}{ Material } & \multicolumn{2}{l|}{ SPB model } & \multicolumn{2}{l|}{ SKB model } \\
\cline { 2 - 5 } & $\mathrm{m}^{*}{ }^{\text {av }}(/ \mathrm{m})$ & $\mu_{0}^{a v}\left(\mathrm{~m}^{2} / \mathrm{Vs}\right)$ & $\mathrm{m}^{*} \mathrm{D}^{\text {av }}(/ \mathrm{m})$ & $\mu_{0}{ }^{a v}\left(\mathrm{~m}^{2} / \mathrm{Vs}\right)$ \\
\hline Mg2Si0.3Sn0.7 & 2.689 & 0.0090 & 3.397 & 0.0118 \\
\hline Mg2Si0.4Sn0.6 & 1.755 & 0.0091 & 2.208 & 0.0125 \\
\hline CoSb3 & 2.787 & 0.0063 & 3.645 & - \\
\hline ZnSb & 0.463 & 0.0295 & 0.589 & 0.0412 \\
\hline PbTe & 0.318 & 0.150 & 0.403 & 0.245 \\
\hline
\end{tabular}

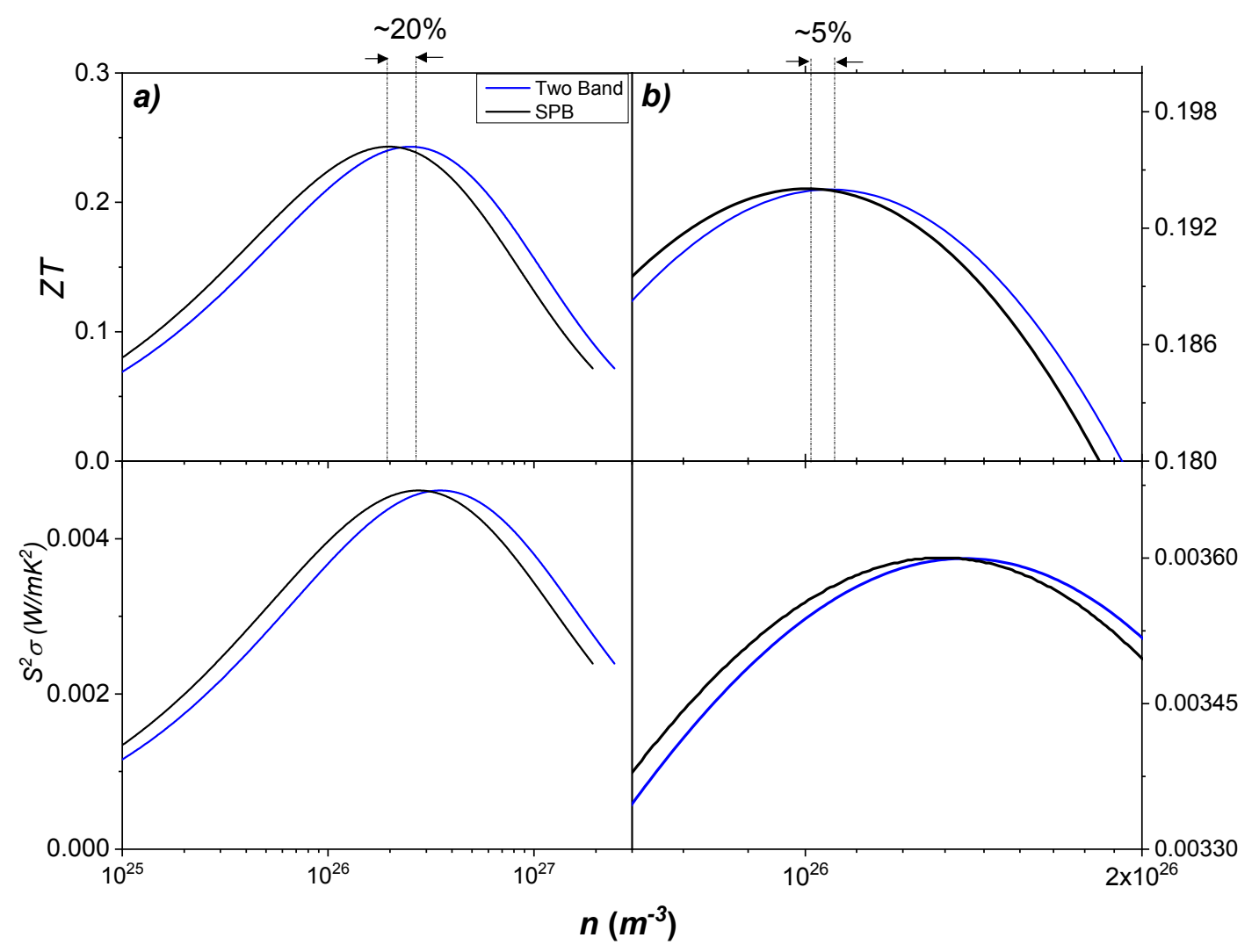

Figure S2: A comparison of errors in the actual $n$ and the $n$ estimated from SPB (with two band generated data as input) as obtained from the $z T$ vs $n$ and $S^{2} \sigma$ vs $n$ plots. The $m^{*}{ }_{D}$ and $\mu_{0}$ values are that of (a) $\operatorname{CoSb}_{3}$ and (b) $\mathrm{Mg}_{2} \mathrm{Si}_{0.3} \mathrm{Sn}_{0.7}$. The errors associated with the SPB $n$ are shown in the figure. 